www.jmscr.igmpublication.org

Index Copernicus Value: 79.54

ISSN (e)-2347-176x ISSN (p) 2455-0450

crossref DOI: https://dx.doi.org/10.18535/jmscr/v7i4.116

\title{
Etio-clinical profile of Cerebral Vein Thrombosis (CVT) Patients in a Tertiary Care Hospital - An Observational Study
}

\author{
Authors \\ Dr Sajad Tak ${ }^{*}$, Dr Sheikh Hilal Ahmad ${ }^{2}$, Dr Atif Rasool Kawoosa ${ }^{3}$ \\ ${ }^{1}$ Senior Resident, Department of General Medicine, GMC Srinagar \\ ${ }^{2}$ Consultant, Department of Neurology, Super speciality Hospital, GMC Srinagar \\ ${ }^{3}$ Senior Resident, Department of Neurology, Super speciality Hospital, GMC Srinagar \\ *Corresponding Author \\ Dr Sajad Tak \\ Email:Taksajad83@gmail.com
}

\begin{abstract}
Background: Cerebral vein thrombosis $(C V T)$ is a rare cerebrovascular disorder with recent increase in recognition due to clinical awareness and easy availability of MRI. CVT has varied clinical presentation and requires high degree of suspicion.CVT usually affects young individuals with annual incidence of 0.22 to 1.57 per 100,000 with female:male ratio of 3:1. The predisposing factors include infectious as well as noninfectious causes with pregnancy and postpartum period being the most common cause among females.

Methodology: The study was an observational hospital based study conducted during a time period of 1 year in General Medicine Department of GMC Srinagar. The study recruited a total of 42 patients who were above 15 years of age and were diagnosed as CVT on the basis of MRI Brain and MRV. The patients were interviewed and their clinical presentation and etiological /predisposing factors were assessed.

Results: in our study, the mean age of the patients was 34.29 years with majority of the patients in the age group of $25-35$ years. The majority of the patients were females with female:male ratio of 2.5:1. The most common presenting symptom was headache in $95.1 \%$ patients followed by seizures in $47.6 \%$ patients. The most common sinus involved was sagittal sinus (38.1\%) followed by equal involvement of sagittal plus transverse sinus and transverse sinus alone (19.1\%). The most common pro-thrombotic condition in our patients was pregnancy and puerperium.

Conclusion: Cerebral vein thrombosis (CVT) is more common in females as compared to males (2.5:1). Any female in pregnancy and puerperium period presenting with seizures and altered sensorium and a woman with history of recent onset headache following intake of OCPs must be evaluated for CVT. All patients suspected for CVT must undergo MRI Brain with MRV for confirmation of diagnosis.

Keywords: Cerebral vein thrombosis, Sagittal sinus, MRV (Magnetic resonance venography), puerpurium.
\end{abstract}

\section{Introduction}

Cerebral vein thrombosis is an uncommon form of stroke usually affecting young individuals. The annual incidence ranges from 0.22 to 1.57 per 100,000 and is more common in female compared to male with the ratio of $3: 1$. It is mainly due to pregnancy, puerperium and use of oral contraceptives. ${ }^{(1)}$ CVT represents $0.5 \%$ to $1 \%$ of all strokes. The diagnosis of CVT is typically based on clinical suspicion and imaging 
confirmation. Clinical findings usually fall in two major categories depending on the mechanism of neurological dysfunction. The first category belongs to those related to increased intracranial pressure attributed to impaired venous drainage. The second category belongs to those related to focal brain damage from venous ischemia / infarction or haemorrhage. The most common presenting symptom is headache mostly secondary to increased intracranial pressure. The headache is typically diffuse and often progresses in severity with passing days. ${ }^{(2)}$

The factors predisposing to CVT can be identified in up to $80 \%$ of patients. There have been numerous conditions which can lead to or predispose to CVT and many a times more than one cause can be in an individual patient. There may be infective as well as non-infective ones. Infective cause may occur following staphylococcal infection of the face. Amongst the non-infective causes, systemic conditions such as connective tissue diseases, other granulomatous or inflammatory disorders and malignancies are most common. $^{(3)}$

The symptoms may include severe headaches, features of raised intracranial tension, seizures, varying degrees of impaired consciousness, cranial nerves impairment, motor and sensory disturbances in the extremities. An obstruction of a major drainage pathway leads to the development of venous intracranial hypertension, cerebral edema, and consequently, CSF hypertension. The ventricles of the brain usually stay at normal size, or become slit-like as a result of the surrounding edema. In the most severe cases, ischemia and/or haemorrhages may develop in the brain tissue. ${ }^{(4)}$

Neuro-imaging is the cornerstone in the diagnosis of cerebral venous sinus thrombosis. Imaging modalities of choice in CVT are CT scan and MRI with MR venogram. CT scan may be normal in $15-30 \%$ cases but MRI with MRV is almost $100 \%$ diagnostic. After introduction of heparin in treatment of CVT mortality has come down significantly and most of the recent studies reporting mortality <20\% compared to earlier studies reporting mortality between 30-50\%. Even though there is a steady decline in the mortality, recent reports still indicate a mortality of 5-30\%. The predictors of poor outcome include: coma, intra-cerebral haemorrhage (ICH), rapidly progressing clinical deficits, posterior fossa lesion and involvement of the deep venous system. ${ }^{(5)}$

\section{Aim and Objectives}

To study the etio-clinical profile of patients having cerebral vein thrombosis attending general medicine department of a tertiary care hospital in northern India.

\section{Methodology}

The study was a hospital based observational study conducted in a tertiary care hospital in Northern India over a period of 1year from October 2017 till October 2018. A total of 42 patients were recruited in the study after taking consent from the patients.

\section{Inclusion criteria}

- Admitted patients in general medicine ward with a diagnosis of CVT

- Age $>15$ years

- Patient and/or relative willing to give consent.

Data were collected using pretested semi-structured questionnaire. Demographic data, clinical features, and risk factors were noted. Clinical examination was done to note focal neurological deficits and mental status. All patients were diagnosed on the basis of MRI brain with MRV. The patients were further assessed for various etiological factors. Data was entered in Excel sheet, and appropriate descriptive statistics were done. For analysis of data SPSS version 20 was used.

\section{Results}

A total of 42 patients were recruited in the study during the study period. The majority of the patients belonged to the age group of $25-35$ years with a mean age of 34.29 years. The 
majority of the patients were females with female to male ratio of 2.5:1. [Figure1].

Figure1: The sex distribution of the patients $(\mathrm{N}=42)$

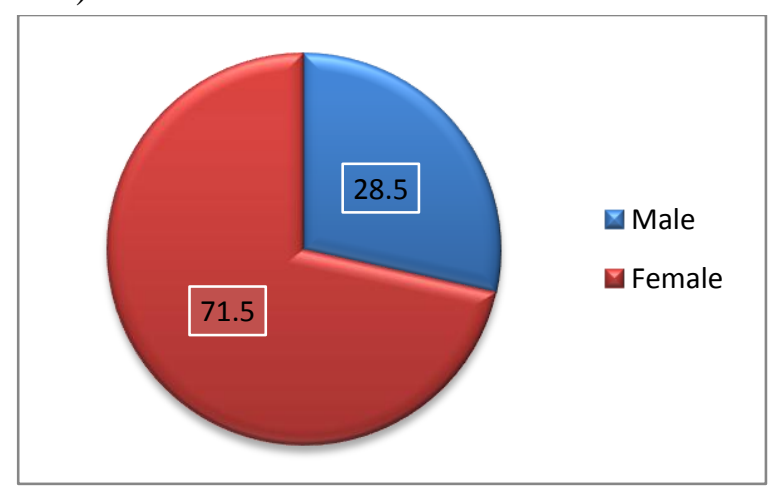

95.2\% patients presented with headache as the main symptom followed by seizures $(47.6 \%)$ and then followed by altered sensorium (16.6\%). [Table1].

Table 1: Distribution of various presenting symptoms in the patients ( $\mathrm{N}=42)$.

\begin{tabular}{|l|c|c|}
\hline Symptom & Number (n) & Percentage (\%) \\
\hline Headache & 40 & 95.2 \\
\hline Seizures & 20 & 47.6 \\
\hline Altered sensorium & 7 & 16.6 \\
\hline Ear discharge & 6 & 14.2 \\
\hline $\begin{array}{l}\text { Focal neurological } \\
\text { deficit }\end{array}$ & 3 & 7.1 \\
\hline $\begin{array}{l}\text { Diminution of } \\
\text { vision }\end{array}$ & 2 & 4.7 \\
\hline Papilledema & 1 & 2.3 \\
\hline Vomiting & 1 & 2.3 \\
\hline
\end{tabular}

On the basis of MRI and MRV, the majority of patients had sagittal sinus thrombosisalone $(38.1 \%)$ followed by sagittal and transverse sinus thrombosis (19\%) with equal incidence of transverse sinus thrombosis alone (19\%).

[Table2].

Table2: Distribution of patients as per the sinus involved $(\mathrm{N}=42)$

\begin{tabular}{|l|c|c|}
\hline Sinus involved & Number (n) & Percentage (\%) \\
\hline Sagittal sinus alone & 16 & 38.1 \\
\hline Sagittal and transverse & 8 & 19.1 \\
\hline Transverse sinus alone & 8 & 19.1 \\
\hline Sigmoid sinus alone & 6 & 14.3 \\
\hline Sagittal and straight & 2 & 4.7 \\
\hline Cavernous sinus alone & 2 & 4.7 \\
\hline
\end{tabular}

The pro-thrombotic conditions in the patients recruited for the study are shown in the table below. (Table3)
Table3: Pro-thrombotic condition associated with the patients studied $(\mathrm{N}=38)$

\begin{tabular}{|l|c|}
\hline Condition & Number(n) \\
\hline Pregnancy and postpartum & 13 \\
\hline CSOM & 6 \\
\hline OCP Intake & 3 \\
\hline Polycythemiarubravera & 2 \\
\hline Malignancy & 2 \\
\hline ANCA positivity & 2 \\
\hline APLA syndrome & 2 \\
\hline Factor V leiden mutation & 2 \\
\hline Protien C and S deficiency & 2 \\
\hline Orbital cellulitis & 2 \\
\hline Anti-thrombin III deficiency & 1 \\
\hline Protein C deficiency alone & 1 \\
\hline
\end{tabular}

*In 4 patients, no pro-thrombotic condition could be ascertained and thus were labelled as idiopathic.

\section{Discussion}

Cerebral venous sinus thrombosis is a rare condition which has been frequently diagnosed due to increased awareness and diagnostic modalities in recent 5 to 10 years $^{(3)}$. The condition is more common in women of reproductive age group mainly attributed to pregnancy, puerperium and OCP use ${ }^{(3)}$.

Our study was a prospective observational hospital based study in which 42 patients were recruited during the time period of 1 year. The patients were examined and interviewed and diagnosis and assessed for various risk factors. The majority of the patients enrolled in the study were in the age group of 25 -35 years with a mean age of 34.29 years. The findings in our study are similar to those studies conducted by Ameri A et $\mathrm{al}^{(6)}$ and Villringer. A et $\mathrm{al}^{(7)}$ and Deepa Dash et al. ${ }^{(8)}$

In our study majority of the patients were females (F:M=2.5:1). The female prepondance was also seen in the studies conducted by Deepa Dash et al ${ }^{(8)}$ and Jam $\operatorname{Stam}^{(9)}$. The female prepondance could be contributed to pregnancy and puerperium being transient prothrombotic states in women. ${ }^{(10)}$

The most common presenting symptom among the patients recruited in our study was headache (95.2\%) followed by seizures (47.6\%) and altered sensorium (16.6\%). These findings were in 
conformity with the studies conducted by Saroja et $\mathrm{al}^{(10)}$ and Amar R Pazare ${ }^{(1)}$. However in the patients with sigmoid sinus thrombosis, ear discharge and deafness were the presenting symptoms.

The main diagnostic modality used in our study was MRI Brain with MRV. The most common sinus involved was sagittal sinus alone $(38.1 \%)$ followed by involvement of sagittal plus transverse sinus (19.1\%). followed by involvement of transverse sinus alone (19.1\%). These findings were consistent with the findings of the studies conducted by Aneesh $\mathrm{T}$ et $\mathrm{al}^{(4)}$ and Khosya $\mathrm{S}^{(5)}$.

The most common pro-thrombotic condition among females was pregnancy and puerperium. This finding is in consistent with the study conducted by Prateek Agarwal et $\mathrm{al}^{(11)}$ and Basawaraj et $\mathrm{al}^{(12)}$. The acquired pro-thrombin conditions were seen in 12 patients $(28.5 \%)$ which is in. conformity with study conducted by Amar R Pazare $^{(1)}$.

\section{Conclusion}

The cerebral vein thrombosis (CVT) is a rare cerebo-vascular disorder that most often affects young adults. The estimated annual incidence being $3-4$ cases per million population ${ }^{(9)}$. About $70 \%$ of adult patients are women.

The clinical features of CVT are highly variable. A woman with history of recent headache after starting OCPs and a woman who presents with seizures and altered sensorium in the postpartum period must be evaluated for CVT.

All the patients with clinical suspicion of CVT should be subjected to MRI Brain with MRV because this is the investigation of the choice as CT Brain may miss the diagnosis.

\section{References}

1. Pazare AR, Karkera KB. Etiology, clinical profile in cortical venous thrombosis. Int $\mathrm{J}$ Adv Med. 2018;5(5):1111-5.
2. STROKE. J Stroke. 2011;42:1158-92.

3. Allroggen H, Abbott RJ. Cerebral venous sinus thrombosis. Postgarduate Med J. 2000;76:12-5.

4. Aneesh T, Gururaj H, Arpitha JS, Rao A, Chakravarthy V, Shetty A. Clinical features , predisposing factors and radiological study of cerebral venous sinus thrombosis : experience from a tertiary care center in Southern India. Int J Res Med Sci. 2017;5(7):3023-8.

5. Khosya S. A Study of Clinical Profile, Risk Factors and Outcome of the Cerebral Venous Sinus Thrombosis ( CVST ): An Experience at a Tertiary Care Center, INDIA. Austin J Neurol Disord Epilepsy. 2018;5(2).

6. Ameri A, Bousser M. Cerebral venous thrombosis. Neurol Clin. 1992;10(1):87-111.

7. Villringer A, Mehraein S, Einhaupl K. Pathophysiological aspects of cerebral venous sinus thrombosis (SVT). J neuroradiol. 1994;21(2):72-80.

8. Dash D, Prasad K, Joseph L. Cerebral venous thrombosis: An Indian perspective. Neurol India. 2015;63(3):318-28.

9. Stam J. Thrombosis of the Cerebral Veins and Sinuses. N Engl J Med. 2005;352:1791-8.

10. Saroja AO, Tapsi C, Karkal NR. Cerebral venous thrombosis in women from Indian subcontinent. Jounal Sci Soc. 2017;44:20-5.

11. Agarwal P, Kumar M, Arora V. Clinical profile of cerebral venous sinus thrombosis and the role of imaging in its diagnosis in patients with presumed idiopathic intracranial hypertension. Indian J Ophthalmol. 2010;(April):153-5.

12. Basavaraj BF, Vandana H. Clinical Profile, Outcome, and Prognostic Factors of Cortical Venous Thrombosis in a Tertiary Care Hospital, India. J Neurosci Rural Pract. 2017;8:204-8. 